\section{N-Myc and STAT Interactor (NMI)}

Brandon J. Metge, Hawley C. Pruitt, Lalita A. Shevde and Rajeev S. Samant

Division of Molecular and Cellular Pathology - Department of Pathology, University of Alabama at Birmingham - Wallace Tumor Institute, Birmingham, AL, USA

\section{Synonyms}

$\mathrm{N}$-myc and STAT interactor; N-myc interactor; NMI

\section{Historical Background}

$\mathrm{N}$-myc interactor, NMI, was first characterized as an interactor of c-Myc and n-Myc using a yeast two-hybrid screen. Since its initial discovery, NMI has been studied for its roles in viral pathologies and more extensively for its role in cancer progression. However, literature describing the role of NMI function in normal physiology is relatively unknown aside from inferences from its roles in various pathologies. NMI is a protein mostly studied for its associations with known transcription factors. In humans, expression profiling has determined that NMI is expressed in all fetal tissues except in the brain and primarily in the adult spleen, liver, and kidneys. The human NMI gene is located on chromosome $2 \mathrm{q} 23$ and contains three exons that can form four alternatively spliced mRNA transcripts; two with no retained introns code for protein products (Bao and Zervos 1996). NMI's expression is largely cytoplasmic; however, it has been detected in the nucleus by multiple studies. Many studies have detailed a growing list of known interactors. As such, the $38 \mathrm{kDa}$ protein has been proposed to be an adapter molecule with different functions depending on cellular context. These proteinprotein interactions occur across all functional domains of NMI. The first hundred amino acids were initially identified to comprise a coiled-coil domain with homology to the $C$. elegans protein CEF59; more recently the $\mathrm{N}$-terminal sequence of NMI is predicted to contain a leucine zipper motif in an alpha-helical configuration similar to that of IFP35 (interferon-inducible protein 35). The NID domain is referred to as an NMI/IFP35 (NID) domain for its homology to IFP35 which is important for protein-protein interactions and cellular localization. The most recent predicted domains are an RNA recognition motif RRM_NMI as well as the RRM_SF, both of which are a part of the RNA-binding motif superfamily (Fig. 1).

\section{NMI Interactors}

NMI effects on cellular function seem to be a result of its various interactions with other molecules most of which are transcription factors. The first evidence of this function was discovered by 


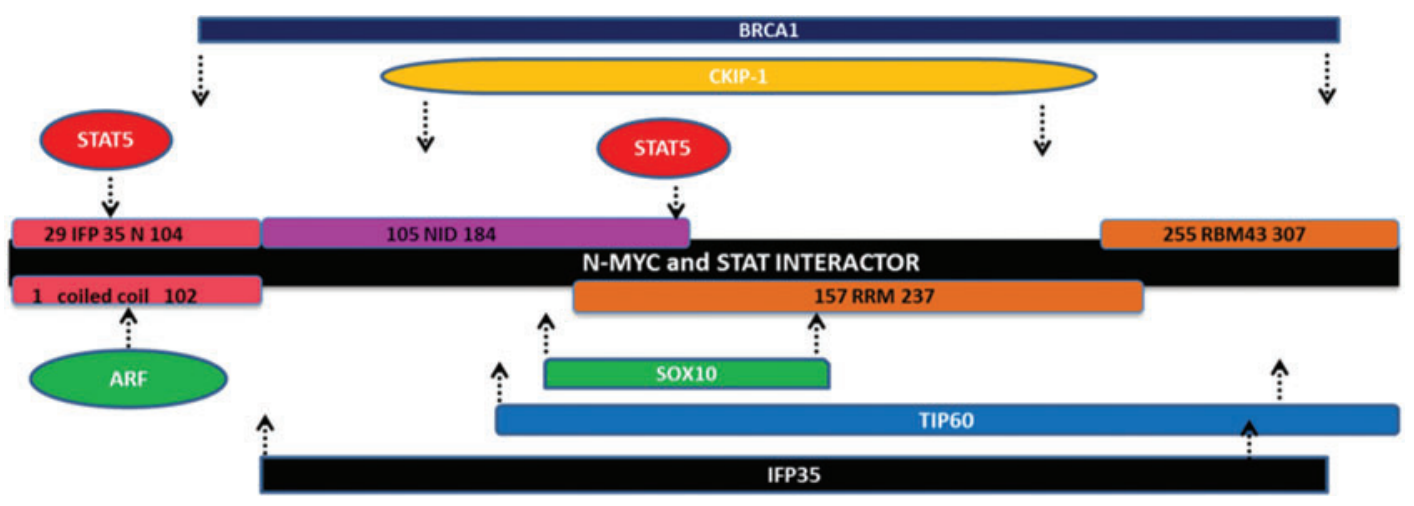

N-Myc and STAT Interactor (NMI), Fig. 1 NMI has multiple functional domains reported to be important for its function. There is an N-terminal domain which has been termed as both the coiled-coil domain and IFP35 domain with highly similar homology to IFP35. The NID domain is thought to be important in protein-protein interaction and

NMI interaction studies demonstrating that NMI binds with all STATs besides STAT2 and phosphorylated or active STAT5b. Furthermore, studies detailing the promoter activity of STAT5 or STAT1 target genes have been shown to be greatly enhanced with NMI interaction (Zhu et al. 1999). Additionally, histone acetyltransferases CBP and p300 have also been implicated as additional binding partners, such that NMI may enhance the interaction between STATs and CBP/p300 (Zhu et al. 1999). NMI was shown to interact with another epigenetic writer, the acetyltransferase Tip60, through multiple domains including its unique NID domain (Zhang et al. 2007). NMI has also been found to be associated with p65 which enhances interaction between p65 and HDACs thereby inhibiting acetylation of p65 and downregulating p65 transcriptional activity (Hou et al. 2016). Moreover, recent studies have further demonstrated NMI as transcriptional regulator of STAT signaling mediated through interactions with heat shock family member Hsp105B. Interaction between NMI and Hsp105B enhanced expression of Hsp70 in a manner dependent on STAT3 (Saito et al. 2014). These studies suggest that NMI recruits epigenetic modifiers and facilitates their binding with transcription factors to enhance transcription of downstream target genes. may be involved in determining cellular localization. Lastly, there are two putative RNA-binding motifs which have yet to be fully characterized. Multiple studies have determined a variety of NMI-interacting proteins most of which have been shown to influence a variety of cellular functions particularly in the context of cancer

Additional evidence for NMI's involvement in modulation of transcription was defined by its interaction in a novel tricomplex between NMI, c-myc, and BRCA1. BRCA1 is essential for maintaining genome stability via its vital roles in DNA double-strand break repair by homologous recombination. BRCA1 is also known to transcriptionally repress another interactor of NMI, MYC. It was demonstrated that alone BRCA1 or NMI did not alter c-Myc transcriptional activation of telomerase reverse transcriptase expression; yet through sequestration of MYC, the complex effectively decreased the transcriptional activity of hTERT (Li et al. 2002).

The discovery of Sox 10 protein as an NMI-binding partner has further demonstrated the importance of NMI as a modulator of transcription factor activity. Sox 10 is well-known for its role in neural crest development and was shown to colocalize with NMI in the nucleus of glioma cell lines. The Sox family of transcription factors is evolutionarily conserved and classified by their HMG box which contains a DNA-binding and DNA-bending domain. They are essential for embryonic developmental processes and control many cell fate decisions including programming of embryonic stem cells. NMI was unable to change Sox10 localization; however, the study showed that NMI increased Sox-mediated 
transcription of its target gene myelin protein zero. Yet, the activity of the dopachrome tautomerase promoter, a second Sox10 target gene that contributes to pigment production in melanocytes, was unchanged by NMI. This suggests that NMI does not act as a global cofactor for transcriptional upregulation, but that genomic context as well as other factors may play a role in dictating NMI's effect on transcription. Moreover, it is more probable that NMI functions as a part of a large protein complex to elicit these functions in combination with Sox 10 as suggested by previous studies with BRCA1 and c-Myc (Schlierf et al. 2005).

As the name suggests, NMI binds to more than just STATs. NMI was discovered in a yeast two-hybrid screen using the bHLH region of NMYC as bait. Further investigation has shown that the bHLH region of NMYC is not necessary for interaction with NMI, and the regions that bind between NMYC and CMYC differ. NMI binds to the DNA-binding region of MYC, while it binds to the acidic central region of the MYCN protein (Bao and Zervos 1996; Bannasch et al. 1999).

Interaction studies have continually demonstrated the role of NMI in stabilization of protein partners within the cytoplasm. NMI was able to block proteasomal degradation of ARF by binding ARF at the same location necessary for ubiquitin ligase interaction. Furthermore, knocking down NMI led to destabilization of ARF. NMI induction upon cellular stress with IFN alpha, cisplatin, or methylmethanesulfate led to its nuclear translocation and stabilization of ARF. NMI overexpression in these cells induced CHK2 activation and G2/M cell cycle arrest especially after DNA damage (Li et al. 2012). In addition to ARF, NMI stabilizes the IFP35 protein through interaction. The N-terminal coiled-coil domain of NMI is necessary, but not sufficient to inhibit proteasomal degradation of IFP35. IFP35 in turn was able to stabilize NMI expression; however, the effect on IFP35 by NMI was more pronounced (Zhou et al. 2000).

\section{Regulation of NMI}

Early studies identified that NMI is regulated at the transcriptional level through induction downstream of inflammatory cytokines IFN $\gamma, \operatorname{IFN} \alpha$, and IL-2. Downstream mediators such as STAT1 and STAT5 are most likely responsible for binding the NMI promoter; furthermore, analysis of the ENCODE data in the USCS portal reveals putative STAT regulatory elements in the NMI sequence. It is interesting that NMI in turn facilitates the activity of these factors allowing for a robust response after cytokine stimulation. Interferon treatment results in high molecular mass complex (HMMC) formation of $300-400 \mathrm{kDa}$, visualized as NMI/IFP35 speckles (NIS), facilitated by the dephosphorylation of IFP35. Other interactors of NMI such as C-Myc, N-Myc, and c-fos are not thought to be contained in the HMMC (Zhou et al. 2000). Molecular analysis confirmed that the fractionation properties of NMI change after treatment with IFN $\gamma$, indicating a shift in subcellular location. Multiple groups have shown that induction of NMI leads to its translocation into the nucleus allowing it to impact STAT-mediated transcription. However, other investigators have found that induction of NMI using cytokines leads to reorganization of NMI protein into puncta within the cytoplasm. Ets-1 was also shown to be an upstream regulator of NMI; it increased mRNA and protein of NMI when expressed in MCF-7 breast cancer cells. The Ets family of transcription factors are highly conserved among species and have documented roles in physiologic and pathologic processes (Jung et al. 2005). Although the Ets motif exists in the NMI gene sequence, the mechanism of upregulation has not been extensively explored; it is likely that it is at the transcriptional level because NMI mRNA expression increased. The study also did not confirm whether Ets-1 directly stimulated expression of NMI; therefore, it is possible that Ets-1 induces the expression of some other factor that in turn upregulates NMI.

Expression profiles of NMI have been most widely studied in the context of disease, namely, cancer. To date studies of NMI have not outlined genetic alterations such as deletions or mutations 
responsible for changes in expression. Most notably, NMI expression is significantly decreased in late stage breast cancer suggesting a transient down-regulation of NMI at the transcript level (Devine et al. 2014). Additionally, NMI expression both at the transcript and protein level has also been shown to be inversely correlated with disease progression in gastric cancers (Hou et al. 2016). Conversely, studies in glioblastoma utilizing three independent data cohorts suggest elevated expression of NMI was linked to poor prognosis and survival. Furthermore, this study was the first to suggest a possible epigenetic regulation of NMI transcript; however, detailed analysis was not preformed to address this possibility (Meng et al. 2015). Studies aimed to specifically address the regulatory networks responsible for modulation of NMI expression demonstrated posttranscriptional regulation of NMI by the micro-RNA family 29 in breast cancer cell lines as well as clinical samples. microRNAs are small noncoding RNAs that target a wide range of mRNA three prime untranslated regions resulting in inhibited translation or mRNA degradation. MiR-29 targeting of the NMI phenocopies previously reports demonstrating various effects on downstream effector pathways (Rostas et al. 2014).

Posttranslational regulation of the NMI protein has not been extensively investigated. The only study to report proteasomal degradation of the NMI protein found that interaction with the severe acute respiratory syndrome coronavirus protein P6 led to ubiquitination of NMI and subsequent degradation (Cheng 2015). NMI is a relatively labile protein with a half-life of approximately $8 \mathrm{~h}$, and this half-life may be extended through interaction with a variety of factors. Zhang and colleagues reported that interaction between Tip60 and NMI in the cytoplasm led to stabilization of the NMI protein. Interaction of the two proteins was not dependent on acyltransferase activity; however, the mechanism of stabilization remains to be discovered as they were unable to show that Tip60 acetylated NMI. They did however show that a different acetyltransferase, p300, acetylated NMI leading to increased levels of expression (Zhang et al. 2007).

\section{Functions in Immunology and Viral Response}

It is intuitive that NMI plays a role in the host's viral response based on its ability to influence JAK/STAT signaling as well as the fact that various cytokines such as IFN $\gamma$, IFN $\alpha$, and IL-2 influence expression of NMI. NMI has been proposed to facilitate apoptosis through interaction with FMDV 2C, a key foot-and-mouth disease viral protein in swine. The formation of protein complexes was dependent on expression of NMI whereby both proteins colocalize within the endoplasmic reticulum. Furthermore, host cell apoptosis was shown to be dependent on NMI expression in FMDV-infected cells (Wang et al. 2012). In other viral studies, NMI was shown to regulate production of interferon in response to viralmediated upregulation of NMI. In these studies, modulation of NMI expression had an inverse effect on interferon production, and Nmi transgenic mice displayed impaired induction of interferon upon viral infection. IRF7, which is a key mediator of this response, was also shown to interact with NMI causing degradation of IRF7 leading to the decreased production of interferon (Wang et al. 2013). Severe acute respiratory syndrome coronavirus protein 6 has also been shown to interact with NMI and mediate proteosomal degradation of NMI thereby halting interferon production and promoting viral survival. Irrespective of effects on interferon signaling, NMI is also able to directly inhibit prototype foamy virus replication. NMI was found to interact with the regulatory protein Tas and sequester it in the cytoplasm inhibiting transactivation of prototype foamy virus' long terminal repeat and internal promoter (Hu et al. 2014). Collectively, these limited studies have suggested an important role for NMI in viral immune response.

\section{NMI Modulation of Cancer Signaling}

Although studies of NMI in the context of normal physiology have been very limited, a wider scope of studies from the perspective of cancer has elucidated various roles of NMI in cancer signaling. 
The role of NMI in modulating the activity of oncogenic transcription factors suggests that NMI is an important protein in cancer development. Some of the first evidence for NMIs involvement in cancer was defined by its interaction in a novel tricomplex between NMI, c-myc, and BRCA1. Through sequestration of MYC, the complex effectively decreased telomerase reverse transcriptase expression, a gene essential for immortalization of cancer cells (Li et al. 2002). A number of studies have been aimed at elucidating the role of NMI in breast cancer. Surveys of breast tumor specimens from the TCGA show little copy number variation or mutation of NMI; however, some independent studies have identified SNPs in the NMI gene present in patient breast cancer as well as ovarian cancer specimens. NMI protein level has been reported to be significantly reduced with increasing stage of breast cancer primary tumors with the lowest expression being seen in patients with metastatic disease. Moreover, NMI mRNA levels decrease with the grade of clinical breast cancer specimens suggesting that loss of NMI facilitates progression to aggressive metastatic disease (Devine et al. 2014).

Metastasis of epithelial cancers is a complex process that employs epithelial-mesenchymal transition (EMT), a developmental phenomenon whereby epithelial cells undergo a change in phenotype allowing them to lose their canonical morphology and invade into surrounding tissue. Silencing NMI in breast cancer cells with an inherent epithelial phenotype causes a morphological change concomitant with increased expression of master EMT regulators ZEB2 and SLUG and subsequent change in EMT marker profiles such as E-cadherin and keratin-18. NMI antagonized TGF $\beta$ signaling, a pathway known to promote EMT, through the upregulation of the inhibitory Smad, Smad7. Through enhancing STAT5-mediated transcription, NMI increased the levels of Smad7 and reversed mesenchymal phenotypes in breast cancer cells (Devine et al. 2014). Negative regulation of EMT in cancer progression via NMI was further solidified by separate work detailing suppression of the NFkB signaling pathway in gastric cancer. NMI expression was found to augment the EMT process in gastric cancer as previously described in breast cancer; however, mechanistically, NMI was found to interact with p65 attenuating NFkB signaling. Exhaustive studies have clearly defined a major role for NFkB in cancer progression and EMT. NFkB signaling is mediated through translocation of $\mathrm{p} 65$ to the nucleus where it functions as a transcription factor. Furthermore, posttranslational modification of p65 is paramount in controlling its transcriptional activity either through differential phosphorylation or acetylation. NMI expression was found to enhance the interaction between p65 and HDACs resulting in decreased acetylation of p65 blocking transcriptional activity ultimately blocking the EMT process (Hou et al. 2016). Together, these studies define a prominent role for NMI as a master regulator of the EMT process via a complex signaling network (Fig. 2).

NMI has been implicated as key regulatory node in the signaling pathways which are major drivers of tumor progression. Some of the earliest reports demonstrated the effect of NMI on Wnt signaling in breast tumor growth. DKK1, the secreted inhibitor of Wnt signaling, was shown to be significantly upregulated by increasing expression of NMI (Fillmore et al. 2009). Independently, DKK1 has been shown to restrict tumor growth in breast cancer. Wnt signaling has roles in the normal physiology of breast development and maturation, but is also seen to be dysregulated in breast cancer. This pathway begins at the cell surface, where Wnt ligands bind to a member of the Frizzled family of receptors and either the LRP5 or LRP6 co-receptor. This tricomplex formation leads to the stabilization of cytosolic $\beta$-catenin, a transcriptional cofactor that can enter the nucleus, bind to a member of the TCF/LEF protein family, and subsequently activate transcription of Wnt target genes (Fig. 2). $\beta$-catenin accumulation is seen in breast carcinomas and is associated with poor prognosis. In breast cancer, deregulation of the Wnt pathway is not due to mutation of the intracellular components as in colorectal cancers. Instead, many breast cancers are augmented at other levels of signaling such as overexpression of LRP6 


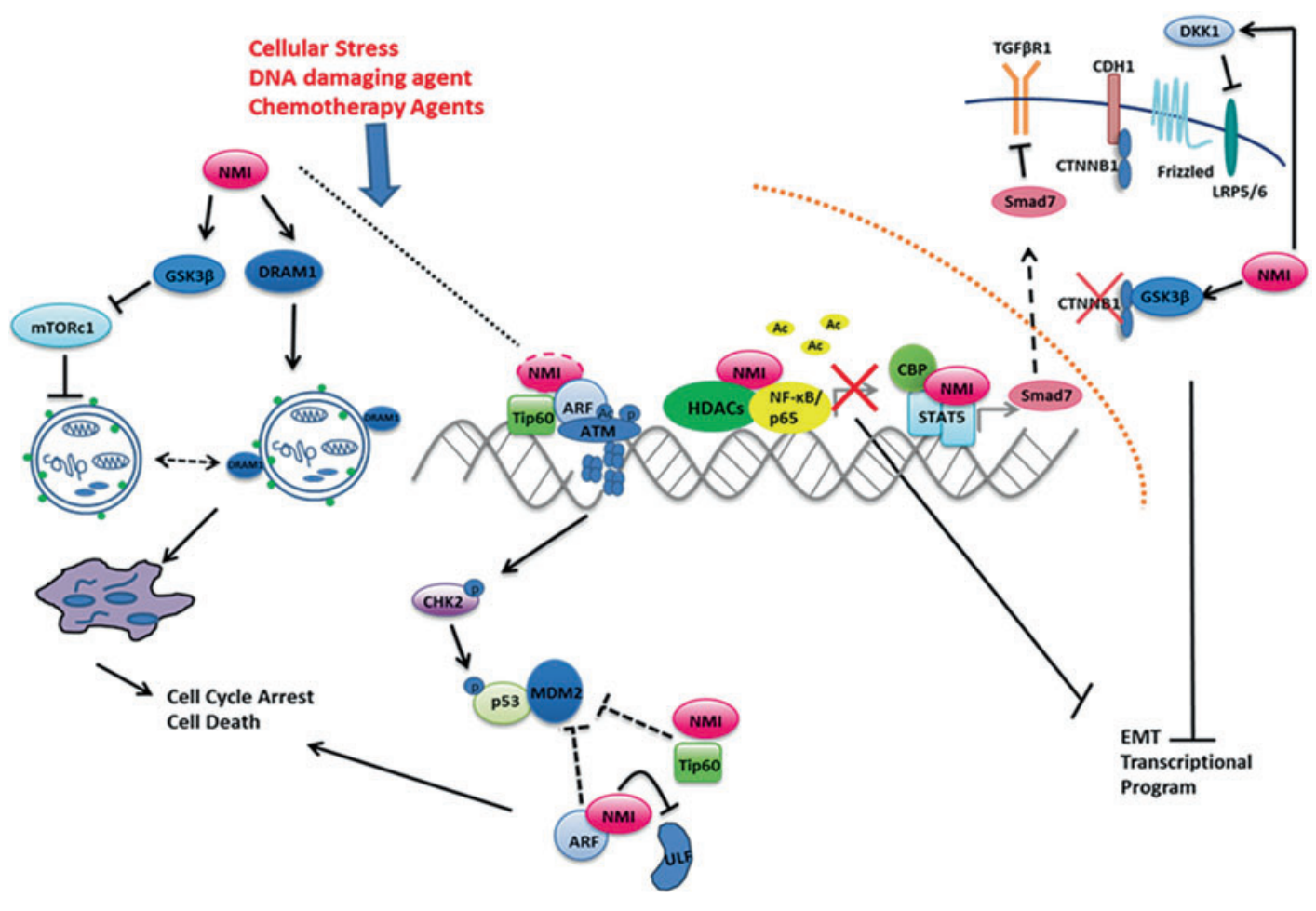

N-Myc and STAT Interactor (NMI), Fig. 2 NMI has broad cellular functions that impact multiple cell signaling pathways and functions. Most of NMI functional impacts have been studied in the context of cancer progression. NMI functions have been linked to multiple signaling pathways, such as Wnt, TGFb, mTOR, and NFkB. NMI has been reported to activate GSK3B and inhibit mTOR signaling as well as upregulate DRAM1, both of which induce autophagic cell death. Furthermore, interactions

co-receptor and the FDZ7 receptor, upregulation of secreted Wnt ligands, and silencing of Wnt inhibitors SFRP-1 and WIF1. Re-expression of NMI in low-expressing breast cancer cell lines lead to increased levels of DKK1 and concomitantly reduced levels of beta-catenin and MYC, known downstream targets of Wnt signaling. In the same study, re-expression of NMI in tumor xenograft models reduced tumor growth in vitro and in vivo as well as invasion and migration of tumor cells (Fillmore et al. 2009). NMI has a multifaceted effect on the Wnt signaling pathway through another interactor, casein kinase 2interacting protein-1 (CKIP-1 or PLEKHO1). CKIP-1 is also able to interact with casein kinase 2 , a protein with noteworthy involvement in the with multiple regulators of the p53/MDM2/ARF pathway allow NMI to functionally impact cell cycle arrest. NMI is also a master regulator of the EMT program through multiple signaling nodes such as NF-KB, Wnt, and TGF $\beta$. NMI interacts with proteins that involved at key regulatory nodes in each of these pathways thereby modifying their functions and ultimately mediating effects on cell death, cell cycle arrest, EMT, and ultimately cancer progression

canonical Wnt signaling pathway. CK-2 phosphorylates and inhibits Dishevelled, a negative regulator of Wnt signaling, and also phosphorylates and stabilizes $\beta$-catenin, which ultimately enhances the transcription of Wnt target genes. How CKIP-1 affects CK-2 and whether its interaction with NMI directly influences Wnt signaling are questions that remain unanswered.

Early studies mostly examined characteristics of NMI in hematologic cells; however, a consistent functional role for NMI in hematologic malignancies has not been identified as of yet. One study discovered that interaction between STAT5, NMI, and N-myc mediates repression of MEF2C leading to increased apoptosis in T-cell acute lymphoblastic leukemia (Nagel et al. 2011). 
Others have identified the apoptosis-inducing protein, apoptin, as yet another interactor of Nmi from a human leukocyte library. Apoptin is encoded by the chicken anemia virus which infects hematopoietic cells in young chickens leading to anemia and eventually cell death. Interestingly, apoptin was found to selectively induce apoptosis in tumorigenic cell lines but not non-transformed non-tumorigenic cells. It was observed that apoptin localized to the nucleus in malignant cells but remained cytoplasmic in normal cells (Nagel et al. 2011). Nuclear localization, through its intrinsic NLS, of apoptin allows it to interact with the anaphase-promoting complex/ cyclosome, causing G2/M cell cycle arrest and apoptosis. Although the nature of the interaction between NMI and apoptin is not well understood, the observation suggests that NMI may play a role in tumor-specific apoptosis.

In contrast to the findings in epithelial cancers, NMI expression in gliomas indicates poor patient prognosis and has been found by one group to independently predict overall survival and progression-free survival. NMI mRNA as well as protein was most highly expressed in stage 4 glioblastoma multiforme, the most common advanced stage of glioma. NMI does not increase in expression with grade; however, GBM has significantly higher expression when compared to normal controls. In vitro and in xenografts, the study found that NMI impacts GBM cell line proliferation through its effect on $\mathrm{G} 0 / \mathrm{G} 1$ cell cycle arrest, and this effect was proposed to be due to NMI binding with STAT1 (Meng et al. 2015). However, it is unclear whether NMI is a driving force behind GBM progression or it is a passenger that provides a good prognostic indicator.

Most recently studies have aimed to examine a role for NMI in mediating cell death in response to chemotherapy in breast cancer. Re-expression of NMI sensitized breast cancer cell lines to DNA-damaging agents such as doxorubicin and cisplatin. Moreover, the role of NMI in sensitization to chemotherapeutic agents was determined to be via induction of autophagy, otherwise termed programmed cell death II. NMI expression in breast cancer cell lines induced autophagic vacuole formation indicative of elevated levels of basal autophagy as determined through enhanced LC3 processing and increased Beclin and p62 expression. Functionally, NMI regulates autophagy by activation of GSK $3 \beta$ which downstream mediates inhibition of mTOR signaling, a known inhibitor of autophagy. In addition to inhibition of mTOR signaling, NMI influences autophagic cell death through upregulation of a vital autophagy component, DRAM1. Silencing of NMI in cancer cell lines was found to dramatically decrease the expression of DRAM1. Additionally, cells silenced for NMI were not able to upregulate DRAM1 in response to cytotoxic stress with cisplatin. Furthermore, knockdown of DRAM1 attenuates the effect of NMI on autophagic cell death mediated in response to chemotherapeutic agents (Fig. 2) (Metge et al. 2015).

Overall, NMI appears to play an essential role in progression or development of a variety of cancers. Loss of NMI in epithelial cancers such as breast cancer leads to unrestricted TGF $\beta$ and Wnt signaling pathways possibly through decreased activity of STAT5 and STAT1, respectively, causing increased invasiveness, growth, and potentially metastasis. Conversely, in cancers that arise from cell types of mesenchymal origin, such as glioblastoma, NMI expression seems to correlate with aggressiveness of disease.

\section{Summary}

NMI is an inducible protein that can be regulated by a variety of cytokines. Initially, described as an interactor of c-Myc and N-Myc as well as STAT, a broader role for NMI in cellular signaling has emerged. Most notably NMI has been found to be important in viral pathologies as well as in cancer. NMI appears to play a prominent role in regulation of multiple cell signaling pathways (Wnt, TGFb, mTOR, NFkB) but also in transcriptional regulation, not as a transcription factor itself, but through its vast protein-protein interaction complexes. Although the most extensive body of work has been done from the perspective of cancer biology, it has become increasingly clear that NMI function spans multiple signaling 
networks and cellular processes. Further studies are needed to unravel the complex cross talk between the various signaling pathways that are modulated by NMI. It will be of continued interest to further studies on this diverse molecule due to its multifaceted involvement in cancer progression and other relevant diseases.

Acknowledgments NIH R01CA194048 grant to R.S.S.

\section{References}

Bannasch D, Weis I, Schwab M. Nmi protein interacts with regions that differ between $\mathrm{MycN}$ and $\mathrm{Myc}$ and is localized in the cytoplasm of neuroblastoma cells in contrast to nuclear MycN. Oncogene. 1999;18(48):6810-7.

Bao J, Zervos AS. Isolation and characterization of Nmi, a novel partner of Myc proteins. Oncogene. 1996;12(10):2171-6.

Cheng W, et al. Severe acute respiratory syndrome coronavirus protein 6 mediates ubiquitin-dependent proteosomal degradation of N-Myc (and STAT) interactor. Virol Sin. 2015;30(2):153-61.

Devine DJ, et al. Loss of N-Myc interactor promotes epithelial-mesenchymal transition by activation of TGF-beta/SMAD signaling. Oncogene. 2014;33(20):2620-8.

Fillmore RA, et al. Nmi (N-Myc interactor) inhibits Wnt/ beta-catenin signaling and retards tumor growth. Int J Cancer. 2009;125(3):556-64.

Hou J, et al. N-Myc-interacting protein (NMI) negatively regulates epithelial-mesenchymal transition by inhibiting the acetylation of NF-kappaB/p65. Cancer Lett. 2016;376(1):22-33.

$\mathrm{Hu} \mathrm{X}$, et al. N-Myc interactor inhibits prototype foamy virus by sequestering viral Tas protein in the cytoplasm. J Virol. 2014;88(12):7036-44.

Jung HH, et al. STAT1 and Nmi are downstream targets of Ets-1 transcription factor in MCF-7 human breast cancer cell. FEBS Lett. 2005;579(18):3941-6.
Li H, Lee TH, Avraham H. A novel tricomplex of BRCA1, Nmi, and c-Myc inhibits c-Myc-induced human telomerase reverse transcriptase gene (hTERT) promoter activity in breast cancer. $\mathrm{J}$ Biol Chem. 2002;277(23):20965-73.

Li Z, et al. NMI mediates transcription-independent ARF regulation in response to cellular stresses. Mol Biol Cell. 2012;23(23):4635-46.

Meng D, et al. High expression of N-myc (and STAT) interactor predicts poor prognosis and promotes tumor growth in human glioblastoma. Oncotarget. 2015;6(7):4901-19.

Metge BJ, et al. N-Myc and STAT Interactor regulates autophagy and chemosensitivity in breast cancer cells. Sci Report. 2015;5:11995.

Nagel S, et al. Transcriptional deregulation of oncogenic myocyte enhancer factor $2 \mathrm{C}$ in T-cell acute lymphoblastic leukemia. Leuk Lymphoma. 2011;52(2):290-7.

Rostas 3rd JW, et al. MicroRNA-29 negatively regulates EMT regulator N-myc interactor in breast cancer. Mol Cancer. 2014;13:200.

Saito Y, et al. Nmi interacts with Hsp105beta and enhances the Hsp105beta-mediated Hsp70 expression. Exp Cell Res. 2014;327(1):163-70.

Schlierf $\mathrm{B}$, et al. The high-mobility group transcription factor Sox 10 interacts with the N-myc-interacting protein Nmi. J Mol Biol. 2005;353(5):1033-42.

Wang J, et al. A critical role of N-myc and STAT interactor (Nmi) in foot-and-mouth disease virus (FMDV) 2C-induced apoptosis. Virus Res. 2012;170(1-2):59-65.

Wang $\mathrm{J}$, et al. Negative regulation of Nmi on virustriggered type I IFN production by targeting IRF7. J Immunol. 2013;191(6):3393-9.

Zhang K, Zheng G, Yang YC. Stability of Nmi protein is controlled by its association with Tip60. Mol Cell Biochem. 2007;303(1-2):1-8.

Zhou X, et al. Interferon-alpha induces nmi-IFP35 heterodimeric complex formation that is affected by the phosphorylation of IFP35. J Biol Chem. 2000;275(28):21364-71.

Zhu M, et al. Functional association of Nmi with Stat 5 and Stat1 in IL-2- and IFNgamma-mediated signaling. Cell. 1999;96(1):121-30. 